Mixing talents
from more than
one field of
chemistry could
provide a blend
of skill-sets
much sought
after in the
increasingly
interdisciplinary
nature of drug
discovery.

Simon Frantz, Associate Editor (News), Nature Reviews Drug Discovery e-mail:s.frantz@nature.com

\section{SHIFTING THE FOCUS OF CHEMISTRY}

In a select band of synthetic organic chemistry laboratories, a regular scenario occurs. A Ph.D. student, usually at an intermediate stage of his or her studies, is distracted from their column by a request from their supervisor: their work to a visitor from one of the leading pharmaceutical companies who is passing through that week?

The visitor has more than just a passing interest in, for example, synthetic routes to a particular natural product. Synthetic organic chemists form the bedrock of chemistry teams in industry (see 'Further reading' in BOX 1). Many leading pharmaceutical companies seeking to fill vacancies share the not unreasonable notion that the most respected and successful synthetic organic chemistry laboratories will craft the most talented synthetic organic chemists. Most chemists are recruited by these companies through visits to the labs of the top synthetic organic chemistry researchers, with requests to see those students deemed to be the best in the group. So-called 'diamonds-in-the-rough' are recruited from other synthetic organic chemistry departments to a lesser degree.

This recruitment strategy has successfully developed good quality chemistry teams devoted to finding highquality compounds that modulate targets of interest. But whether these teams can be improved is being increasingly questioned. Some chemists in academia and industry argue that the present approach creates pockets of self selection. Like recruits like, which results in a relatively narrow team focus in tackling the chemistry of drug discovery. It might also discourage a student from diversifying experiments in the remainder of their studies. could they do a short 15-minute-or-so presentation of
One suggestion for improvement is to consider mixing chemistry talents, say the critics. Adding innovative thinkers from other chemistry backgrounds to a core synthetic organic chemistry team could ensure that issues are analysed and addressed in a more comprehensive manner. Experience in pursuing research in more than one discipline could provide the talents much sought after in response to the increasingly interdisciplinary nature of drug discovery.

If companies can be persuaded by this viewpoint, the winners are likely to be chemical biologists. These researchers have, in general, been ignored by the pharmaceutical industry because companies prefer researchers with extensive, focused experience rather than broader, but less in-depth, experience.

But companies are always trying to make the drug discovery and development process more effective and efficient; for example, in response to the need to develop small molecule modulators for novel targets that are emerging from the human genome sequence. This is increasingly requiring integration of chemistry and biology knowledge.

However, most biologists do not have the chemistry knowledge to pick the right kinds of compounds to test in their assays, and most chemists cannot pick the right assays with which to test their compounds. Stories from academia claiming that some companies, for the first time, have recruited chemical biologists in the last 12 months, suggests that the industry could be beginning to examine ways of fostering constructive discussion between groups that have traditionally suffered from lack of communication.

\section{Box 1 | Websites and further reading}

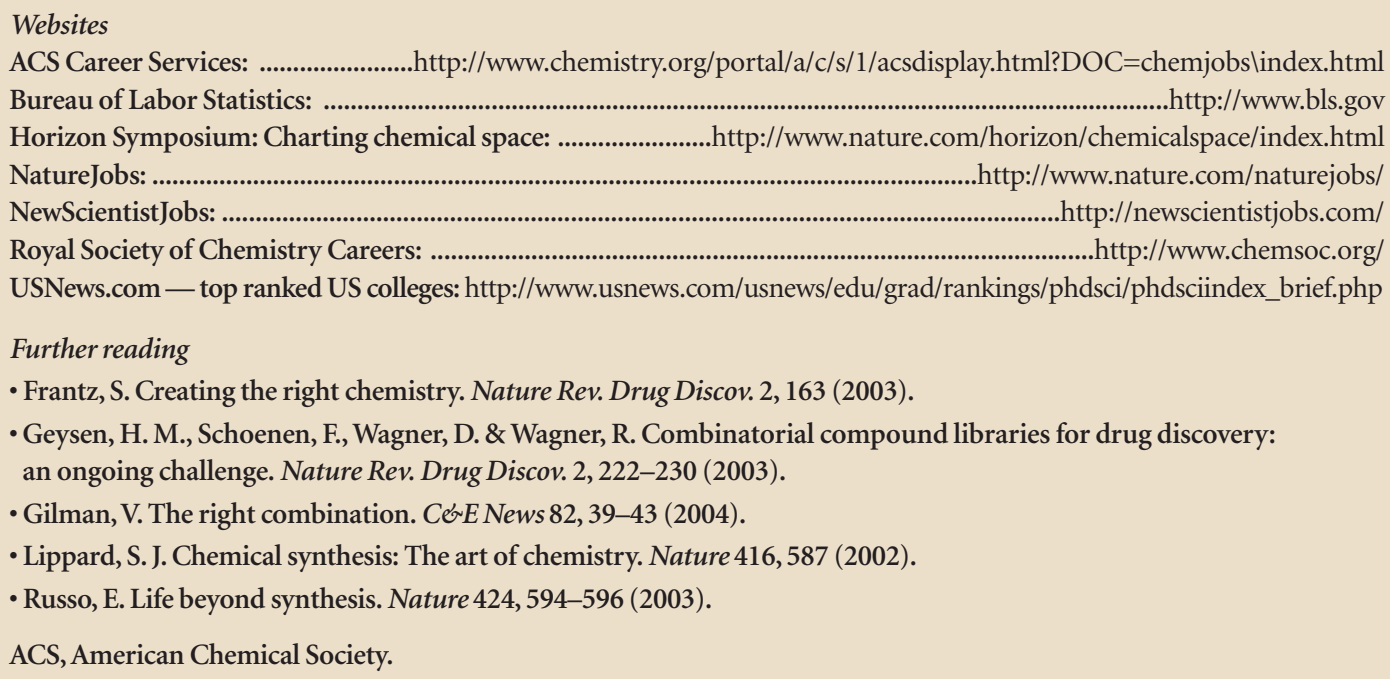

